\title{
Fish scale is a suitable model for analyzing determinants of skeletal fragility in type 2 diabetes
}

\author{
Nobuo Suzuki ${ }^{1} \cdot$ Kei-ichiro Kitamura ${ }^{2} \cdot$ Atsuhiko Hattori $^{3}$
}

Received: 3 October 2016 / Accepted: 17 October 2016 / Published online: 28 October 2016

(C) Springer Science+Business Media New York 2016

The teleost fish has a unique hard tissue, scales that consist of osteoblasts (bone formation cells), osteoclasts (bone resorption cells), and calcified bone matrix, including type 1 collagen, bone $\gamma$-carboxyglutamic acid protein, osteonectin, and hydroxyapatite [1-6]. In all vertebrates, including teleosts, blood calcium levels are strictly kept at a constant concentration $(2 \sim 3 \mathrm{mM})$ between each species [7]. Teleost scales have an important function in regulating blood calcium levels because teleost scales, having both osteoblasts and osteoclasts, known to function as potential internal calcium reservoirs similar to those in the endoskeletons of mammals $[2,5,8]$. Particularly, in fresh water teleosts such as zebrafish and goldfish, which live in a low-calcium environment, their scales play a very important role in regulating the plasma calcium level.

In fish scales, as well as bone, several calcemic hormones function in osteoblasts and osteoclasts. In the scales of goldfish, parathyroid hormone (PTH), a hypercalcemic hormone, acts on osteoblasts and then activated osteoclasts via receptor activators of nuclear factor- $\mathrm{\kappa B}$ (RANK)/ receptor activators of the nuclear factor- $\mathrm{\kappa B}$ ligand

Nobuo Suzuki

nobuos@staff.kanazawa-u.ac.jp

1 Noto Marine Laboratory, Institute of Nature and Environmental Technology, Kanazawa University, Noto-cho, Ishikawa 927-0553, Japan

2 Department of Clinical Laboratory Science, Graduate School of Medical Science, Kanazawa University, Kanazawa, Ishikawa 9200942, Japan

3 Department of Biology, College of Liberal Arts and Sciences, Tokyo Medical and Dental University, Ichikawa, Chiba 272-0827, Japan
(RANKL) system, as PTH does in mammalian osteoblasts and osteoclasts [9]. The hypocalcemic hormone, calcitonin, suppressed osteoclastic activity in the scales of goldfish $[10,11]$. Furthermore, in zebrafish, arachidonic acid, which is metabolized by cyclooxygenases to prostaglandin $E_{2}$ $\left(\mathrm{PGE}_{2}\right.$ ), affected scale regeneration (following the removal of ontogenetic scales) and reduced mineral deposition resulting from the activation of matrix metalloproteinase 2 and 9 (osteoclastic markers) [12]. In goldfish, $\mathrm{PGE}_{2}$ acts on osteoblasts and then increases the osteoclastic activity in the scales of goldfish via the RANK-RANKL system [13]. The increase of RANKL (osteoclastogenesis-promoting factor) mRNA expression by $\mathrm{PGE}_{2}$ treatment induced hypercalcemia in goldfish [13]. In addition to the above hormonal responses, the morphological features of osteoclasts in teleost scales are very similar to those of mammalian osteoclasts. Multinucleated osteoclasts with large numbers of mitochondria and lysosomes were observed on the pit of the scale $[9,14]$. Some osteoclasts were attached to the scale by a well-developed ruffled border [9, 14]. Judging from observations of scale regeneration, the morphological features of osteoblasts were very similar to the development of mammalian membrane bone [15]. Teleost scales have a number of physiological and morphological features in common with mammalian bone.

Diabetes mellitus (DM), commonly referred to as diabetes, is a group of metabolic diseases that are characterized by chronic hyperglycemia. DM is classified as being caused by either the pancreas not producing enough insulin (type 1 DM: T1DM) or the cells (muscle, fat, and other cells) of the body not responding properly to the insulin produced (type 2 DM: T2DM) [see a review, 16]. Furthermore, DM induces other pathologies, including bone disease, diabetic retinopathy, nephropathy, neuropathy, cardiomyopathy, and 
microangiopathy [16]. In the case of bone disease, T1DM leads to a high risk of fracture and osteoporosis because the bone mineral density (BMD) is reduced in T1DM [16]. However, it has been debated whether T2DM is associated with high risk of fractures even in presence of normal or low-normal BMD [17]. To analyze the detailed mechanism of the relationship between T2DM and bone disease, a suitable model is earnestly desired.

In the study by Carnovali et al. [18], the authors introduced a new model for studying glucose and bone metabolism. Using the scales of zebrafish, a suitable model, Carnovali et al. [18] examined the effect of hyperglycemia on bone metabolism. After 28 days of treatment with $4 \%$ $(w / v)$ glucose concentrations, the morphology of the retinal blood vessels showed abnormalities resembling human diabetic retinopathy. Next, bone metabolism was evaluated using scales stained with calcein. Due to osteoclastic bone resorption, a reduction (12.4\%) of the scale area was measured on the surface of the scales in hyperglycemic zebrafish. The osteoporotic-like phenotype was visualized through the mineral matrix staining of scales. In addition, hyperglycemic zebrafish scales showed a significant decrease of osteoblastic marker enzyme (alkaline phosphatase) activity and an increase of osteoclastic marker enzyme (tartrate-resistant acid phosphatase) activity. The expression of osteoblastic marker mRNAs, such as osteocalcin and osteoprotegerin, decreased significantly. However, further study is needed to analyze RANKL (osteoclastogenesispromoting factor) mRNA expression at several time courses because RANKL mRNA expression in the scales of hyperglycemic zebrafish decreased significantly.

Our recent data [19] was supported by the conclusions of Carnovali et al. [18]. Namely, after a 2-week exposure to hyperglycemia, the high molecular weight fractions of bone matrix collagen increased in the regenerating scales of hyperglycemic goldfish, as compared with those of euglycemic (control) goldfish. This suggests that non-enzymatic cross-linking by advanced glycation end products (AGEs) was occurring in the regenerating scales of hyperglycemic goldfish. Thereafter, we conducted an in vitro study with collagen extracted from goldfish scales. As a result, we demonstrated that glyceraldehyde-driven non-enzymatic cross-linking of collagen fibril compositions (particularly $\gamma$ fraction) quickly increased with glyceraldehyde treatment. This change in the molecular size of collagen fibrils indicated the formation of multiple AGEs via a non-enzymatic condensation reaction between reducing sugars and amino groups of lysine or arginine residues of collagen fibril protein. The findings of our in vitro study regarding glyceraldehyde-derived AGEs are in agreement with findings in diabetic rats [20].

Taking these results into consideration, we conclude that the scales of teleosts such as zebrafish and goldfish are a suitable model of hyperglycemic disease and will help elucidate the molecular mechanisms of bone metabolic changes in human diabetic patients.

Funding This study was supported in part by grants to N.S. (Grantin-Aid for Scientific Research [C] No. 16K07871 by JSPS), to K.K. (Grant-in-Aid for Scientific Research [C] No. 15K01705 by JSPS), and to A.H. (Grant-in-Aid for Scientific Research [C] No. 24570068 by JSPS).

\section{Compliance with ethical standards}

Conflict of interest The authors declare that they have no competing interests.

Ethical approval This article does not contain any studies with human participants performed by any of the authors.

\section{References}

1. H. Onozato, N. Watabe, Studies on fish scale formation and resorption III: fine structure and calcification of the fibrillary plates of the scales in Carassius auratus (Cypriniformes: Cyprinidae). Cell. Tissue. Res. 201, 409-422 (1979)

2. J. Bereiter-Hahn, L. Zylberberg, Regeneration of teleost fish scale. Comp. Biochem. Physiol. A 105, 625-641 (1993)

3. N. Suzuki, A. Hattori, Melatonin suppresses osteoclastic and osteoblastic activities in the scales of goldfish. J. Pineal. Res. 33, 253-258 (2002)

4. B. Redruello, M.D. Estevao, J. Rotllant, P.M. Guerreiro, L.I. Anjos, A.V.M. Canario, D.M. Power, Isolation and characterization of piscine osteonectin and down regulation of its expression by PTH-related protein. J. Bone. Miner. Res. 20, 682-692 (2005)

5. N. Suzuki, M. Somei, A. Seki, R.J. Reiter, A. Hattori, Novel bromomelatonin derivatives as potentially effective drugs to treat bone diseases. J. Pineal. Res. 45, 229-234 (2008)

6. T.A. Thamamongood, R. Furuya, S. Fukuba, M. Nakamura, N. Suzuki, A. Hattori, Expression of osteoblastic and osteoclastic genes during spontaneous regeneration and autotransplantation of goldfish scale: a new tool to study intramembranous bone regeneration. Bone 50, 1240-1249 (2012)

7. P.M. Guerreiro, J.L. Renfro, D.M. Power, A.V.M. Canario, The parathyroid hormone family of peptides: structure, tissue distribution, regulation, and potential functional roles in calcium and phosphate balance in fish. Am. J. Physiol. Regul. Integr. Comp. Physiol. 292, R679-R696 (2007)

8. P. Persson, K. Sundell, B.Th Björnsson, H. Lundqvist, Calcium metabolism and osmoregulation during sexual maturation of river running Atlantic salmon. J. Fish. Biol. 52, 334-349 (1998)

9. N. Suzuki, J.A. Danks, Y. Maruyama, M. Ikegame, Y. Sasayama, A. Hattori, M. Nakamura, M.J. Tabata, T. Yamamoto, R. Furuya, K. Saijoh, H. Mishima, A.K. Srivastav, Y. Furusawa, T. Kondo, Y. Tabuchi, I. Takasaki, V.S. Chowdhury, K. Hayakawa, T.J. Martin, Parathyroid hormone 1 (1-34) acts on the scales and involves calcium metabolism in goldfish. Bone 48, 1186-1193 (2011)

10. N. Suzuki, T. Suzuki, T. Kurokawa, Suppression of osteoclastic activities by calcitonin in the scales of goldfish (freshwater teleost) and nibbler fish (seawater teleost). Peptides 21, 115-124 (2000)

11. T. Sekiguchi, N. Suzuki, N. Fujiwara, M. Aoyama, T. Kawada, K. Sugase, Y. Murata, Y. Sasayama, M. Ogasawara, H. Satake, Calcitonin in a protochordate, Ciona intestinalis: the prototype of 
the vertebrate Calcitonin/Calcitonin gene related peptide superfamily. FEBS. J. 276, 4437-4447 (2009)

12. E. de Vrieze, M. Moren, J.R. Metz, G. Flik, K.K. Lie, Arachidonic acid enhances turnover of the dermal skeleton: studies on zebrafish scales. PLoS. One. 9, e89347 (2014)

13. K. Omori, S. Wada, Y. Maruyama, A. Hattori, K. Kitamura, Y. Sato, M. Nara, H. Funahashi, K. Yachiguchi, K. Hayakawa, M. Endo, R. Kusakari, S. Yano, A.K. Srivastav, T. Kusui, S. Ejiri, W. Chen, Y. Tabuchi, Y. Furusawa, T. Kondo, Y. Sasayama, T. Nishiuchi, M. Nakano, T. Sakamoto, N. Suzuki, Prostaglandin $\mathrm{E}_{2}$ increases both osteoblastic and osteoclastic activities in the scales of goldfish and participates in the calcium metabolism in goldfish. Zool. Sci. 29, 499-504 (2012)

14. K. Azuma, M. Kobayashi, M. Nakamura, N. Suzuki, S. Yashima, S. Iwamuro, M. Ikegame, T. Yamamoto, A. Hattori, Two osteoclastic markers expressed in multinucleate osteoclasts of goldfish scales. Biochem. Biophys. Res. Commun. 362, 594-600 (2007)

15. H. Yoshikubo, N. Suzuki, K. Takemura, M. Hoso, S. Yashima, S. Iwamuro, Y. Takagi, M.J. Tabata, A. Hattori, Osteoblastic activity and estrogenic response in the regenerating scale of goldfish, a good model of osteogenesis. Life Sci. 76, 2699-2709 (2005)

16. Y.-Y. Wu, E. Xiao, D.T. Graves, Diabetes mellitus related bone metabolism and periodontal disease. Int. J. Oral Sci. 7, 63-72 (2015)

17. K. Wongdee, N. Charoenphandhu, Update on type 2 diabetesrelated osteoporosis. World J. Diabetes 6, 673-678 (2015)

18. M. Carnovali, L. Luzi, G. Banfi, M. Mariotti, Chronic hyperglycemia affects bone metabolism in adult zebrafish scale model. Endocrine. doi:10.1007/s12020-016-1106-3

19. K. Kitamura, T. Andoh, W. Okesaku, Y. Tazaki, K. Ogai, K. Sugitani, I. Kobayashi, N. Suzuki, W. Chen, M. Ikegame, A. Hattori, Effects of hyperglycemia on bone metabolism and bone matrix in goldfish scales. Comp. Biochem. Physiol. A. 203:152-158 (2016)

20. Y. Kitahara, M. Takeuchi, K. Miura, T. Mine, T. Matsui, S. Yamagishi, Glyceraldehyde-derived advanced glycation end products (AGEs): a novel biomarker of postprandial hyperglycaemia in diabetic rats. Clin. Exp. Med. 8, 175-177 (2008) 